\title{
Novas curvas de crescimento da Organização Mundial da Saúde: comparação com valores de crescimento de crianças pré-escolares das cidades de Taubaté e Santo André, São Paulo
}

New World Health Organization growth standards: comparison with growth data of preschool children of the cities of Taubaté and Santo André, São Paulo, Brazil

Claudio Leone ${ }^{1}$, Ciro João Bertoli ${ }^{2}$, Denise de Oliveira Schoeps ${ }^{3}$

\section{RESUMO}

Objetivo: Em 2007, a Organização Mundial da Saúde (OMS) propôs um referencial de crescimento de cinco a 19 anos, a ser utilizado em continuidade ao referencial de menores de cinco anos (de 2006), que, entre outras características, aos cinco anos de idade mostra um bom ajuste com a curva de 2006. Este estudo visa comparar os referenciais da OMS com o crescimento observado em pré-escolares brasileiros.

Métodos: A partir dos dados antropométricos de 2.830 crianças com idade entre três e seis anos de duas amostras das cidades de Taubaté e Santo André, em São Paulo, foram calculados os valores dos percentis (P) 5, 50 e 95 de peso, estatura e índice de massa corpórea (IMC). Os valores dos novos referenciais da OMS foram comparados a esses resultados de acordo com o sexo e a faixa etária.

Resultados: Nos percentis de estatura, o referencial apresentou valores próximos ou pouco superiores aos dos pré-escolares até os cinco anos. Nessa idade, a tendência se inverte, obtendo-se valores progressivamente inferiores até os sete anos. Para peso e IMC, em todas as idades consideradas, o P5 e 50 dos referenciais são pouco menores que os das crianças, mas o P95 indica uma tendência de crescimento progressivamente menor, fazendo com que, próximo aos sete anos, o P95 de IMC dos pré-escolares da amostra seja cerca de $4 \mathrm{~kg} / \mathrm{m}^{2}$ maior.

Conclusões: Os referenciais da OMS apontam uma prevalência menor de magreza (desnutrição) e baixa estatura entre cinco e sete anos e, ao mesmo tempo, uma elevação importante da prevalência de obesidade entre três e sete anos de idade.

Palavras-chave: crescimento; pré-escolares; antropometria; valores de referência; Organização Mundial da Saúde.

\section{ABSTRACT}

Objective: The World Health Organization (WHO), in 2007, proposed a growth reference standard from five to 19 years to be used in continuation to the 2006 one (for children younger than five years-old). This growth chart shows a satisfactory adjustment at five years with the 2006 curve. This study aims to compare WHO references with the growth observed in Brazilian preschool children.

Methods: 2,830 children between three and six years old from Taubaté and Santo André cities, in São Paulo State, Brazil, were studied regarding weight, height and body mass index (BMI). The $5^{\text {th }}, 50^{\text {th }}$ and $95^{\text {th }}$ percentiles $(\mathrm{P})$ were calculated in several ages for both genders and compared to the WHO reference values.

Results: Concerning height, the WHO reference values were closer to or a little higher than the Brazilian studied samples until five years. At this time, an opposed tendency was noticed with WHO height percentiles becoming progressively below the Brazilian samples until the age of seven. For weight and BMI in all considered ages, the $5^{\text {th }} \mathrm{P}$ and the $50^{\text {th }} \mathrm{P}$ of the WHO referential were slightly lower than the children's ones, but the $95^{\text {th }} \mathrm{P}$ shows a trend of a progressively lower growth close to seven years: the BMI
Instituição: Faculdade de Saúde Pública da Universidade de São Paulo (FSP/USP), São Paulo, SP, Brasil, Universidade de Taubaté (Unitau), Taubaté, SP, Brasil, e Faculdade de Medicina do ABC (FM-ABC), Santo André, SP, Brasil

'Professor-associado do Departamento de Saúde Materno-Infantil da FSP/ USP, São Paulo, SP, Brasil

2Professor-assistente, Doutor da Disciplina de Pediatria do Departamento de Medicina da Unitau, Taubaté, SP, Brasil

${ }^{3}$ Mestre em Ciências e professora-assistente do Departamento MaternoInfantil da FM-ABC, Santo André, SP, Brasil
Endereço para correspondência:

Claudio Leone

Avenida Doutor Arnaldo, 715, sala 218

CEP 01246-904 - São Paulo/SP

E-mail: leone.claudio@gmail.com

Recebido em: 22/7/08

Aprovado em: 28/9/08 
$95^{\text {th }} \mathrm{P}$ from the preschool samples were around $4 \mathrm{~kg} / \mathrm{m}^{2}$ higher than WHO values.

Conclusions: The use of WHO reference growth chart will produce a lower prevalence of malnutrition and short stature between five and seven years and, at the same time, an important rise of obesity prevalence between ages three and seven.

Key-words: growth; child, preschool; anthropometry; reference values; World Health Organization.

\section{Introdução}

Com a finalidade de fornecer um bom referencial para monitorar o crescimento de crianças e adolescentes que também pudesse ser útil para a avaliação nutricional, a Organização Mundial da Saúde (OMS), em 2006 e 2007 $7^{(1,2)}$, colocou à disposição dos profissionais de saúde dois conjuntos de tabelas e gráficos de evolução, de acordo com a idade e o sexo, de peso, comprimento, estatura, índice de massa corpórea (IMC) e outros parâmetros: as denominadas curvas de crescimento da OMS.

O primeiro conjunto, de 2006, abarca o período do nascimento até os cinco anos de idade. Seus dados são provenientes de um estudo original multicêntrico, de diferentes grupos étnicos, com metodologia adequada e envolvendo uma grande amostra de crianças que receberam aleitamento materno de maneira compatível com o padrão mínimo recomendado pela própria OMS em 1996, data de planejamento do estudo. Essas curvas foram propostas pela OMS como prescritivas, isto é, correspondentes ao melhor padrão de crescimento, do ponto de vista biológico, que deveria ser esperado em todas as crianças ${ }^{(1)}$. Como a monitoração do crescimento é um processo contínuo até o final da adolescência, a utilização desse novo referencial gerou a necessidade de definir qual seria o padrão mais adequado para continuar o acompanhamento do crescimento após os cinco anos de idade.

Em 2007, com o propósito de resolver essa questão, a própria OMS publicou um novo conjunto de curvas elaboradas a partir de um referencial anterior, proposto em 2000 pelos Centers for Disease Control (CDC) dos Estados Unidos, cujos dados foram revistos e reprocessados de maneira a atenuar os problemas anteriormente detectados com sua utilização. Essas modificações processadas pela OMS nos dados do CDC resultaram em um referencial que apresenta um ajuste bastante adequado, com pequenas diferenças em valor absoluto, com os valores da Curva da OMS 2006 para os cinco anos de idade e que, ao final da adolescência, tem pontos de corte para definir alterações de índice de massa corpórea (IMC) próximos aos aceitos para adultos jovens ${ }^{(2-5)}$.

Atualmente, no Brasil, considerando os cuidados metodológicos adotados na sua confecção, os referenciais da OMS, além de serem referendados por diversas sociedades científicas, também já vêm sendo utilizados por instituições de saúde públicas e privadas, o que enfatiza a necessidade de comparar as tendências de crescimento propostas pelas curvas da OMS com dados reais de nossa população, como já tem sido feito para outras populações ${ }^{(4-8)}$.

Partindo destas premissas, procedeu-se à comparação da tendência de crescimento evidenciada pelos referenciais propostos pela OMS com a tendência de crescimento apresentada por um conjunto de crianças brasileiras em idade pré-escolar, de modo a abranger a junção entre os dois referenciais da OMS (2006 e 2007) aos cinco anos de idade. Embora haja um ajuste aparentemente satisfatório, como refere a $\mathrm{OMS}^{(2)}$, esse ponto representa um momento de junção de dados procedentes de duas populações completamente distintas e, portanto, demanda novas avaliações daquilo que ocorre durante o uso do referencial de 2007 em continuidade ao de 2006.

\section{Métodos}

O presente trabalho utiliza dados secundários provenientes de dois estudos anteriores, de desenho transversal, que realizaram um levantamento de valores de crescimento de crianças pré-escolares e cujas amostras foram reunidas em um único conjunto de estudo, com o qual se compararam os valores dos referenciais da OMS.

Agruparam-se dois conjuntos de crianças de três anos completos a sete anos incompletos que frequentavam creches e pré-escolas em dois municípios do estado de São Paulo, Taubaté e Santo André, e cujos dados de crescimento (peso e estatura) foram obtidos em dois estudos de corte transversal, realizados entre 2001 e 2006 , perfazendo um total de 3.101 crianças.

O primeiro conjunto, de Santo André, incluiu o universo de crianças pré-escolares que frequentavam as 14 creches filantrópicas existentes na cidade e vinculadas à Federação das Entidades Assistenciais de Santo André (Feasa) ${ }^{(9)}$ e o segundo conjunto é uma amostra casual probabilística, por conglomerados, de pré-escolares que frequentavam regularmente as 50 creches e pré-escolas públicas municipais de Taubaté(10).

Ambos os estudos anteriores haviam obtido, a partir do prontuário existente nas unidades de ensino, as informa- 
ções relativas a: identificação, data de nascimento, sexo e morbidade. Para fins desta análise, excluíram-se as crianças que, por ocasião da avaliação antropométrica, não estavam presentes ou que apresentavam antecedentes de morbidade capazes de comprometer seu crescimento (doenças crônicas ou específicas do crescimento).

Embora fossem duas amostras de municípios diferentes e também pertencentes a níveis socioeconômicos distintos, os dois grupos apresentavam um padrão de crescimento estatural muito semelhante, em média praticamente igual ao proposto pelo referencial do Centers for Diseases Control and Prevention dos Estados Unidos ${ }^{(9,10)}$. Como o objetivo era comparar o novo referencial da OMS a valores de crianças brasileiras que estivessem crescendo de maneira aparentemente satisfatória, optou-se pela fusão das duas amostras em uma única, de modo a obter maior precisão dos valores estimados, já que isso praticamente dobrava o tamanho da amostra.

Das 3.101 crianças que restaram no grupo de estudo após a triagem por morbidade, calculou-se a idade decimal em anos, excluindo-se, em seguida, os dados de 247 crianças que estavam fora da faixa etária de interesse no presente estudo, isto é, tinham menos de três anos ou aquelas com sete anos ou mais de idade. Excluíram-se dados de 24 crianças ( $0,8 \%$ do total) cujos valores apresentavam inconsistência entre a antropometria e a idade calculada, utilizando-se como ponto de corte uma estatura para sexo e idade e/ou um peso para estatura e idade acima ou abaixo de quatro desvios padrão (dp) em relação à média do grupo.

Todas as crianças cuja avaliação foi incluída neste estudo estavam, por ocasião das pesquisas iniciais, regularmente matriculadas nas creches e pré-escolas. Dos pré-escolares, $1.461(51,6 \%)$ eram do sexo masculino e $1.369(48,4 \%)$ do sexo feminino. A distribuição por faixa etária é apresentada na Tabela 1, que evidencia uma distribuição por idade muito semelhante para ambos os sexos.

Nos estudos iniciais, os pré-escolares foram pesados e tiveram sua estatura medida na própria instituição de ensino.
A realização dessas mensurações foi efetuada por profissionais de saúde ligados à própria instituição ou ao projeto de pesquisa em andamento devidamente treinados e que utilizavam técnicas e instrumental propostos internacionalmente para essa finalidade ${ }^{(11)}$, sempre sob supervisão de pediatras e/ou nutricionistas do grupo de pesquisa. Todos os autores participaram da execução e/ou da supervisão de pelo menos um dos levantamentos antropométricos.

$\mathrm{Na}$ ocasião, todas as crianças foram pesadas e medidas sem sapatos e com o mínimo de roupa possível, sendo os valores obtidos registrados imediatamente após a tomada em planilha especificamente elaborada para a pesquisa. Na realização das medidas, contou-se com a colaboração das educadoras e professoras das respectivas salas, de maneira a se obter uma maior colaboração das crianças.

Após a coleta de peso e estatura, os dados foram armazenados em planilha do programa Microsoft ${ }^{\circledR}$ Excel e calculou-se o índice de massa corpórea $\left(\mathrm{IMC}=\mathrm{kg} / \mathrm{m}^{2}\right)$ de cada criança. Para este estudo, após verificar a consistência dos dados, calculou-se a média, o desvio padrão, a mediana, os percentis (P) 5 e 95 de estatura, peso e IMC por sexo e grupo de idade das 2.830 crianças pré-escolares. Os intervalos de idade considerados para os cálculos foram de seis meses, estimando-se os valores para a mediana de idade das crianças incluídas em cada intervalo.

Os valores absolutos das curvas da OMS de 2006 e 2007 para estatura, peso e IMC, correspondentes aos percentis 5, 50 e 95, foram comparados graficamente com os valores calculados dos pré-escolares de Taubaté e Santo André para o mesmo sexo e idade.

\section{Resultados}

As Tabelas 2, 3 e 4 mostram os valores calculados da mediana, dos percentis 5 e 95 de peso, estatura e IMC dos pré-escolares, por sexo e faixa de idade. As Figuras 1 a 6 mostram a evolução de estatura, peso e IMC de meninos e

Tabela 1 - Distribuição da amostra de pré-escolares segundo idade e sexo

\begin{tabular}{lccr}
\hline Faixa etária & $\begin{array}{c}\text { Sexo masculino } \\
\mathbf{n}(\%)\end{array}$ & $\begin{array}{c}\text { Sexo feminino } \\
\mathbf{n}(\%)\end{array}$ & \multicolumn{1}{c}{$\begin{array}{c}\text { Total } \\
\mathbf{n}(\%)\end{array}$} \\
\hline 3 a $<$ de 4 anos & $183(12,5)$ & $156(11,4)$ & $339(11,9)$ \\
4 a $<$ de 5 anos & $366(25,1)$ & $354(25,9)$ & $720(25,5)$ \\
5 a $<$ de 6 anos & $472(32,3)$ & $444(32,4)$ & $916(32,4)$ \\
6 a $<$ de 7 anos & $440(30,1)$ & $415(30,3)$ & $855(30,2)$ \\
Total & $\mathbf{1 . 4 6 1 ( 1 0 0 )}$ & $\mathbf{1 . 3 6 9 ( 1 0 0 )}$ & $\mathbf{2 . 8 3 0 ( 1 0 0 )}$ \\
\hline
\end{tabular}


Tabela 2 - Valores de peso em P5, P50 e P95, em kg, segundo sexo e idade, dos pré-escolares

\begin{tabular}{lccc|ccc}
\hline \multicolumn{4}{c|}{ Meninos } & \multicolumn{3}{c}{ Meninas } \\
\hline $\begin{array}{l}\text { Idade } \\
\text { (anos) }\end{array}$ & $\begin{array}{c}\text { Percentil 5 } \\
(\mathbf{k g})\end{array}$ & $\begin{array}{c}\text { Percentil 50 } \\
\mathbf{( k g )}\end{array}$ & $\begin{array}{c}\text { Percentil 95 } \\
\mathbf{( k g )}\end{array}$ & $\begin{array}{c}\text { Percentil 5 } \\
\mathbf{( k g )}\end{array}$ & $\begin{array}{c}\text { Percentil 50 } \\
\text { (kg) }\end{array}$ & $\begin{array}{c}\text { Percentil 95 } \\
\text { (kg) }\end{array}$ \\
\hline 3 a 3,49 & 12,0 & 14,7 & 18,0 & 11,8 & 14,6 & 18,3 \\
3,5 a 3,99 & 13,0 & 16,0 & 19,8 & 12,6 & 15,7 & 19,9 \\
4 a 4,49 & 13,7 & 16,8 & 20,9 & 13,3 & 16,6 & 21,2 \\
4,5 a 4,99 & 14,1 & 18,0 & 22,5 & 14,0 & 17,7 & 22,7 \\
5 a 5,49 & 15,3 & 18,9 & 23,5 & 14,9 & 18,6 & 23,9 \\
5,5 a 5,99 & 16,1 & 19,9 & 25,0 & 15,6 & 19,6 & 25,4 \\
6 a 6,49 & 17,0 & 21,1 & 26,6 & 16,4 & 20,7 & 27,0 \\
6,5 a 6,99 & 17,8 & 22,1 & 27,9 & 17,2 & 21,8 & 28,6 \\
\hline
\end{tabular}

Tabela 3 - Valores de estatura em P5, P50 e P95, em centímetros, segundo sexo e idade, dos pré-escolares

\begin{tabular}{lccc|ccc}
\hline \multicolumn{5}{c|}{ Meninos } & \multicolumn{3}{c}{ Meninas } \\
\hline $\begin{array}{l}\text { Idade } \\
\text { (anos) }\end{array}$ & $\begin{array}{c}\text { Percentil 5 } \\
(\mathbf{c m})\end{array}$ & $\begin{array}{c}\text { Percentil 50 } \\
\text { (cm) }\end{array}$ & $\begin{array}{c}\text { Percentil 95 } \\
(\mathbf{c m})\end{array}$ & $\begin{array}{c}\text { Percentil 5 } \\
\text { (cm) }\end{array}$ & $\begin{array}{c}\text { Percentil 50 } \\
\text { (cm) }\end{array}$ & $\begin{array}{c}\text { Percentil 95 } \\
\text { (cm) }\end{array}$ \\
\hline 3 a 3,49 & 91,1 & 97,4 & 103,6 & 91,2 & 97,7 & 104,3 \\
3,5 a 3,99 & 95,4 & 102,2 & 109,0 & 94,6 & 101,5 & 108,5 \\
4 a 4,49 & 97,9 & 105,0 & 112,1 & 97,2 & 104,5 & 111,8 \\
4,5 a 4,99 & 101,4 & 108,9 & 116,4 & 100,2 & 107,8 & 115,5 \\
5 a 5,49 & 103,7 & 111,3 & 119,0 & 102,7 & 110,6 & 118,6 \\
5,5 a 5,99 & 106,5 & 114,5 & 122,4 & 105,4 & 113,7 & 121,9 \\
6 a 6,49 & 109,2 & 117,4 & 125,7 & 108 & 116,6 & 125,1 \\
6,5 a 6,99 & 111,3 & 119,8 & 128,3 & 110,5 & 119,4 & 128,2 \\
\hline
\end{tabular}

Tabela 4 - Valores de Índice de Massa Corpórea em P5, P50 e P95, em kg/m², segundo sexo e idade, dos pré-escolares

\begin{tabular}{lccc|ccc}
\hline \multicolumn{5}{c|}{ Meninos } & \multicolumn{3}{c}{ Meninas } \\
\hline $\begin{array}{l}\text { Idade } \\
\text { (anos) }\end{array}$ & $\begin{array}{c}\text { Percentil 5 } \\
\left(\mathbf{k g} / \mathbf{m}^{2}\right)\end{array}$ & $\begin{array}{c}\text { Percentil 50 } \\
\left(\mathbf{k g} / \mathbf{m}^{2}\right)\end{array}$ & $\begin{array}{c}\text { Percentil 95 } \\
\left(\mathbf{k g} / \mathbf{m}^{2}\right)\end{array}$ & $\begin{array}{c}\text { Percentil 5 } \\
\left(\mathbf{k g} / \mathbf{m}^{2}\right)\end{array}$ & $\begin{array}{c}\text { Percentil 50 } \\
\left(\mathbf{k g} / \mathbf{m}^{2}\right)\end{array}$ & $\begin{array}{c}\text { Percentil 95 } \\
\left(\mathbf{k g} / \mathbf{m}^{2}\right)\end{array}$ \\
\hline 3 a 3,49 & 13,7 & 15,5 & 17,8 & 13,4 & 15,3 & 17,8 \\
3,5 a 3,99 & 13,5 & 15,4 & 17,6 & 13,2 & 15,3 & 17,8 \\
4 a 4,49 & 13,4 & 15,3 & 17,6 & 13,2 & 15,3 & 17,9 \\
4,5 a 4,99 & 13,3 & 15,2 & 17,6 & 13,1 & 15,3 & 18,0 \\
5 a 5,49 & 13,4 & 15,3 & 17,7 & 13,1 & 15,2 & 18,1 \\
5,5 a 5,99 & 13,4 & 15,3 & 17,8 & 13,1 & 15,3 & 18,3 \\
6 a 6,49 & 13,4 & 15,3 & 17,9 & 13,1 & 15,3 & 18,5 \\
6,5 a 6,99 & 13,5 & 15,4 & 18,1 & 13,1 & 15,4 & 18,7 \\
\hline
\end{tabular}




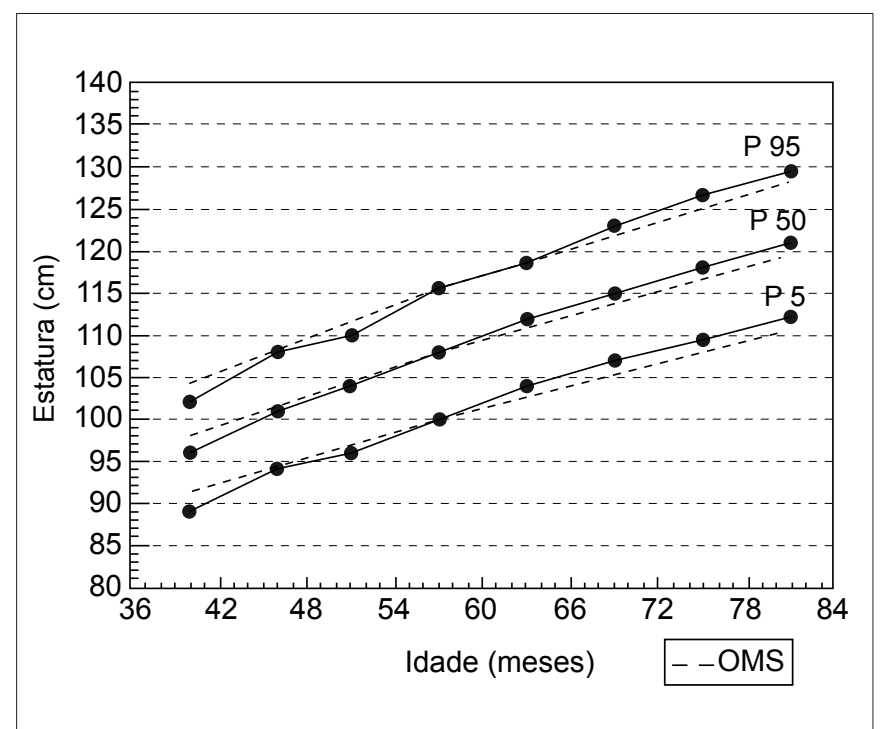

Figura 1 - Comparação da estatura de meninas pré-escolares de Taubaté e Santo André com os valores das curvas da Organização Mundial de Saúde de 2006 e 2007.

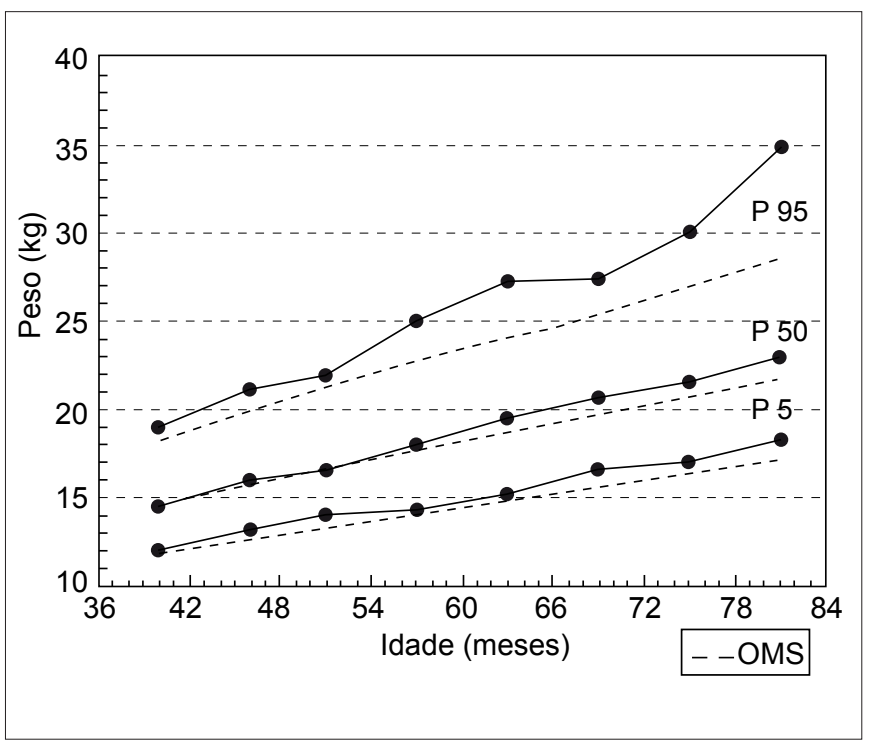

Figura 3 - Comparação da estatura de meninas pré-escolares de Taubaté e Santo André com os valores das curvas da Organização Mundial de Saúde de 2006 e 2007.

meninas, segundo a idade, em comparação com os valores dos referenciais propostos pela OMS em 2006 e 2007.

As tabelas e gráficos dos resultados evidenciam uma tendência de evolução, em valores absolutos, muito semelhante para ambos os sexos em cada parâmetro estudado. Observa-se nos gráficos que o percentil 95 de peso e consequentemente de IMC propostos pela OMS tendem a alcançar, com a idade, valores absolutos muito menores do que os valores do grupo de pré-escolares brasileiros, o que praticamente não se observa

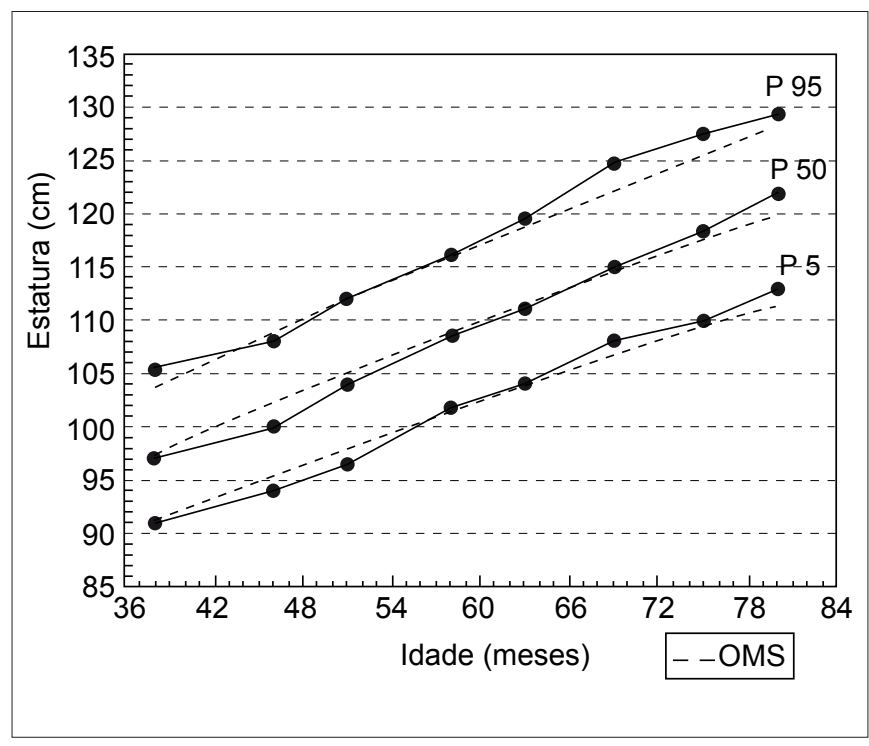

Figura 2 - Comparação da estatura de meninos pré-escolares de Taubaté e Santo André com os valores das curvas da Organização Mundial de Saúde de 2006 e 2007.

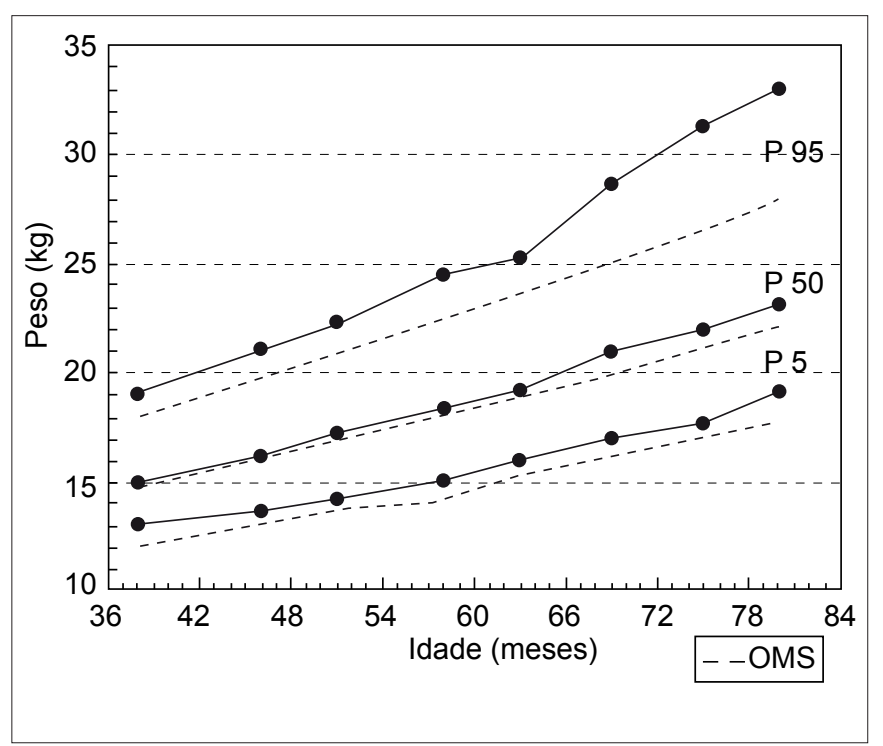

Figura 4 - Comparação da estatura de meninos pré-escolares de Taubaté e Santo André com os valores das curvas da Organização Mundial de Saúde de 2006 e 2007.

para a evolução da estatura. Para a mediana e para o percentil 3 de peso e de IMC, os valores absolutos do referencial OMS também são menores do que os correspondentes dos pré-escolares. Porém, a diferença é muito menor do que a observada para o P95.

Quanto à estatura, observam-se duas fases distintas: uma primeira com valores absolutos do referencial de 2006 um pouco superiores, que se inverte a partir dos cinco anos, quando os valores das crianças brasileiras passam a ser 


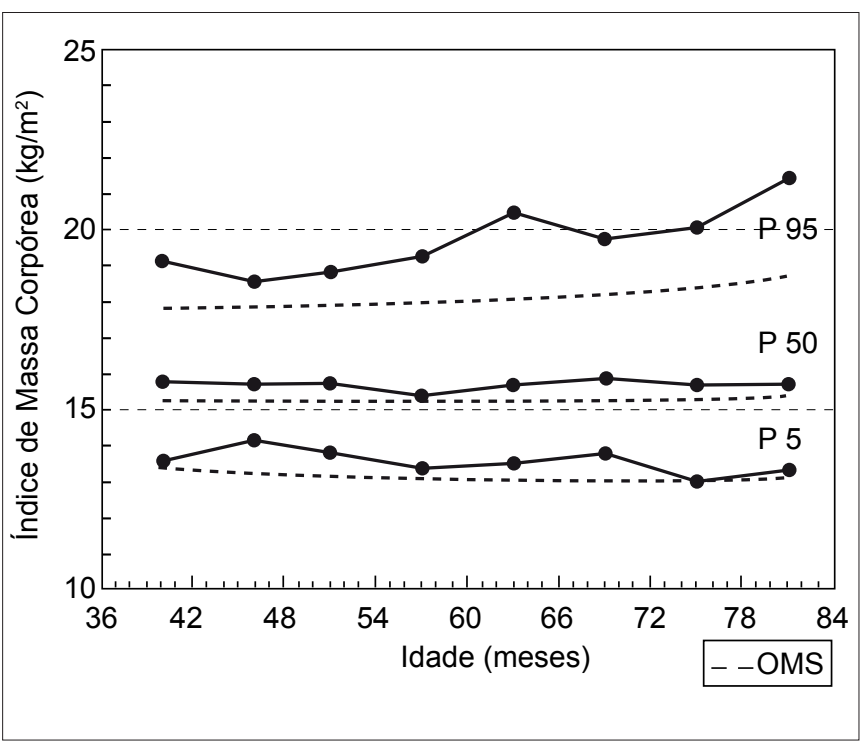

Figura 5 - Comparação do Índice de Massa Corpórea de meninas pré-escolares de Taubaté e Santo André com os valores das curvas da Organização Mundial de Saúde de 2006 e 2007.

progressivamente maiores, mesmo que com uma diferença pequena, que os valores do referencial da OMS de 2007, nos três percentis comparados.

\section{Discussão}

Apesar de a OMS afirmar que o peso, a estatura e o IMC aos 60 meses pouco diferem em valores absolutos entre os dois referencias ${ }^{(2)}$, é possível que os valores de peso e estatura nos anos imediatamente antes e após esse ponto não reflitam uma mesma tendência de crescimento. A reconstrução da curva para os maiores de cinco anos com base nos dados já existentes do CDC, mesmo que revistos de modo a excluir os valores realmente extremos (os denominados outliers) e da revisão do modelo matemático aplicado para suavizar os valores reais, não permite pressupor, a priori, que realmente se trate agora de um referencial adequado ${ }^{(2,3)}$.

Aliás, algumas restrições se aplicam também à curva de 2006 da OMS, como a definição do pré-requisito de aleitamento materno adotado para os dois grupos estudados: o longitudinal e o transversal, que foi diferente, muito mais exigente para o grupo em seguimento longitudinal. A questão da representatividade da amostra também não é totalmente desprezível, talvez seja a melhor possível, mas, por exemplo, não contempla a etnia amarela e, tecnicamente, é uma amostra de conveniência, com suas implicações na validade externa e, portanto, na sua representatividade. Isso é

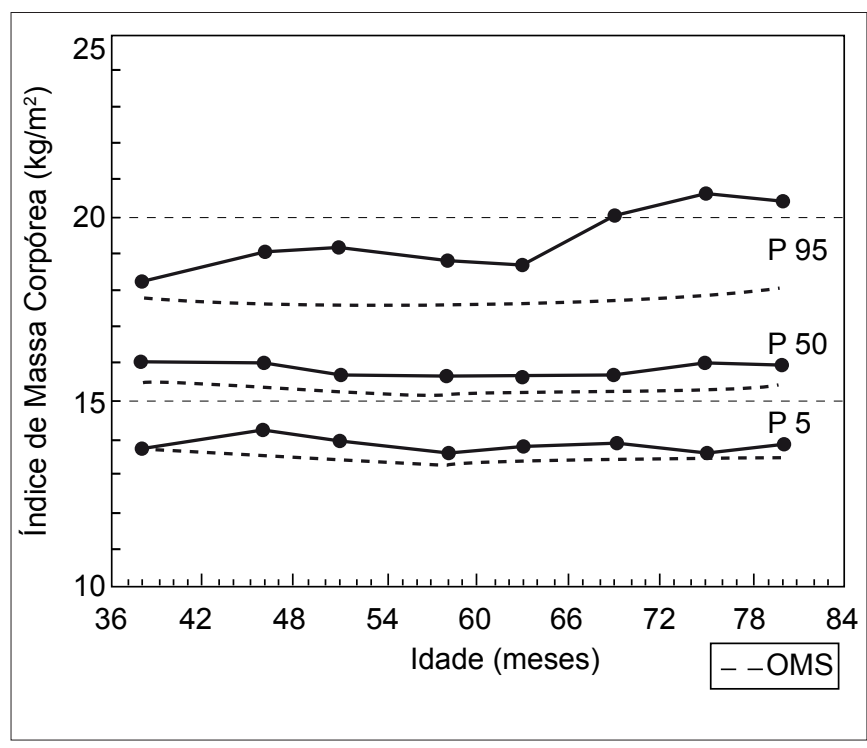

Figura 6 - Comparação do Índice de Massa Corpórea de meninos pré-escolares de Taubaté e Santo André com os valores das curvas da Organização Mundial de Saúde de 2006 e 2007.

agravado ainda mais pelas perdas ocorridas durante o estudo, que no grupo longitudinal ficaram por volta de $50 \%{ }^{(2)}$.

O que se pode dizer é que, embora ambos os referenciais tenham superado diversos problemas metodológicos existentes em referenciais anteriormente propostos, tecnicamente, no senso mais estrito do termo, não podem ser considerados padrões universais de crescimento. Ele podem, sim, ser os referenciais de melhor qualidade disponíveis no momento para monitorar o crescimento nos países que não têm um referencial próprio ou que, mesmo que o tenham, este não seja muito adequado do ponto de vista metodológico.

Todas essas considerações fazem com que as comparações dos dois referenciais propostos pela OMS com grupos de crianças de diferentes origens sejam de particular relevância para o entendimento da forma como os mesmos se situam quando utilizados em distintas populações; visão crítica esta que é de fundamental importância para sua utilização quase indiscriminada, como é a proposta da OMS.

A comparação pode ser ainda mais relevante quando abarca a faixa etária que engloba o ponto de junção entre os dois referenciais da OMS, ou seja, ao redor dos cinco anos de idade. Sua importância se destaca ao se considerar que, na monitoração individual do crescimento, os dois referenciais são utilizados como se fizessem parte de um continuum, o que poderia levar a conclusões diferentes quanto ao crescimento de uma mesma criança, conforme a idade considerada. Se os 
dois referenciais não fossem compatíveis na idade de junção, o seu emprego na rotina da atenção básica de saúde poderia ser comprometido.

A comparação da evolução da estatura dos três aos seis anos completos de idade entre os referenciais e os valores observados em pré-escolares das duas cidades brasileiras mostra que há dois comportamentos distintos: o primeiro em que o referencial apresenta valores superiores aos dos pré-escolares, com tendência a aproximar-se dos mesmos, e o segundo período, após os 60 meses, no qual os valores do referencial OMS se tornam progressivamente inferiores aos das crianças.

Essa evolução é muito similar nos três percentis que foram comparados e para ambos os sexos. Preliminarmente, tal evolução poderia ser atribuída ao fato de se tratar de dois referenciais diferentes. Entretanto, como explicação alternativa, ou que se soma à anterior, há o fato de que o referencial OMS até cinco anos de idade é resultante do estudo de uma população de "elite", diferente da população de Taubaté e de Santo André com crianças de famílias de classe socioeconômica média, média baixa ou baixa ${ }^{(9,10)}$. A diferença de nível socioeconômico, entretanto, não explica o fato de os valores praticamente se igualarem aos 60 meses de idade, a não ser que ocorresse um progressivo catch up no crescimento das crianças de menor renda, o que é pouco provável, considerando-se que se trata de crianças em idade pré-escolar.

Estudo recente, que comparou o crescimento de duas coortes de crianças inglesas ao referencial da OMS de 2006, evidenciou que, no período de seguimento dos dois aos cinco anos de idade, o crescimento médio de estatura alcançado pelas 2.258 crianças monitoradas situava-se abaixo dos valores do referencial. Uma das duas coortes (1.335 crianças) mostrou uma tendência de redução nessa diferença, sem, no entanto, conseguir igualar o referencial e atingindo, entre os quatro e cinco anos de idade, um escore $\mathrm{Z}$ médio de estatura de $-0,11^{(3)}$.

Se a explicação residisse apenas no catch up de crescimento, seria de se esperar que, uma vez alcançado o mesmo valor do referencial, o catch up cessasse, fazendo com que, a partir desse ponto, o crescimento assumisse a mesma velocidade. Entretanto, não é o que se observou, pois após os 60 meses de idade, o segundo referencial da OMS (2007) mantém sistematicamente valores abaixo dos da amostra populacional que é proveniente de um estrato socioeconômico provavelmente mais baixo, qualquer que seja o percentil analisado. Isso permitiria pressupor que o referencial OMS 2007 evi- dencia um crescimento estatural menor do que o potencial de crescimento de crianças brasileiras nessa faixa de idade, pelo menos as incluídas neste estudo.

A comparação do peso e, simultaneamente, do IMC entre o referencial e os dados das crianças das duas cidades estudadas mostra que os valores do referencial são nitidamente menores praticamente em todas as idades, nos três percentis analisados e em ambos os sexos. Nesses parâmetros antropométricos, o percentil 95 mostrou a diferença mais acentuada, diferença esta que tende a aumentar com a idade, fazendo com que, por volta dos sete anos de idade, alcance, em valores absolutos, uma diferença de seis quilos ou mais no peso, resultando em P95 de IMC cerca de $4 \mathrm{~kg} / \mathrm{m}^{2}$ menor no referencial da OMS, em ambos os sexos. Um estudo das duas coortes de crianças inglesas também mostrou valores de peso e, principalmente, de IMC sistematicamente superiores aos do referencial entre dois e cinco anos de idade ${ }^{(3)}$.

O conjunto dessas diferenças poderia ser interpretado como consequência de comportamentos diversos entre as populações no que se refere ao aleitamento materno, já que, por definição metodológica, as crianças do estudo da OMS devem ter tido um melhor padrão de aleitamento do que aquele que se costuma observar nas populações em geral, o que teria um efeito protetor sobre o excesso de peso posterior $^{(12-14)}$. Entretanto, a mesma explicação não se adequaria à evolução do percentil 95 após os 60 meses. O mais provável é que essa diferença seja decorrente das características que o novo referencial de 2007 apresenta, em função da decisão metodológica de excluir as crianças portadoras de peso e IMC extremamente elevados (outliers) da amostra utilizada para desenvolver o modelo das curvas, o que resulta em redistribuição dos percentis do referencial que define menores valores absolutos ${ }^{(2)}$.

Do ponto de vista epidemiológico, a transição nutricional observada de maneira quase universal ${ }^{(15)}$ pode explicar, pelo menos parcialmente, os valores maiores observados nas amostras aqui estudadas bem como os das coortes de crianças inglesas, já que, independentemente do padrão de aleitamento materno, tem-se observado um deslocamento da tendência ao excesso de peso para idades cada vez menores e a prevalência de sobrepeso e obesidade com uma evolução ascendente com a idade, já na faixa etária pré-escolar ${ }^{(16)}$.

De qualquer maneira, independentemente de possíveis explicações para as diferenças encontradas entre os referenciais da OMS e os dados das crianças de Taubaté e Santo André, as conclusões preliminares dessas análises são que, ao se adotarem os novos referenciais para a avaliação de crianças 
em idade pré-escolar entre três e sete anos incompletos, é preciso ter em mente os seguintes aspectos:

1. Para o crescimento em estatura, aparentemente não há maiores problemas quanto à utilização do referencial de 2006. Entretanto, após os cinco anos de idade, há o risco de subestimar, mesmo que com uma diferença não muito grande em valores absolutos, o potencial de crescimento, com a utilização do referencial de 2007.

2. Para a evolução do peso e do IMC, duas situações ocorrem:

- com a utilização, como ponto de corte, dos valores relativos aos percentis do limite inferior da curva de distribuição, o novo referencial privilegia a especificidade (identificando apenas os casos de maior magreza) em detrimento da sensibilidade (excluindo, portanto, crianças ainda em risco potencial de subnutrição). Embora a subnutrição não seja tão prevalente em nosso meio, como ocorre em outros países afetados pela Transição Nutricional ${ }^{(17)}$, ainda está presente;

- com a utilização dos valores de correspondentes aos percentis superiores de distribuição de peso e IMC da OMS para avaliar o excesso de peso (sobrepeso ou obesidade) em pré-escolares, o referencial privilegia

\section{Referências bibliográficas}

1. Garza C. New growth standards for the 21st century: a prescriptive approach. Nutr Rev 2006;64:S55-9.

2. de Onis M, Onyango AW, Borghi E, Siyam A, Nishida C, Siekmann J. Development of a WHO growth reference for school-aged children and adolescents. Bull World Health Organ 2007;85:660-7.

3. Butte NF, Garza C, de Onis M. Evaluation of the feasibility of international growth Standards for school-aged children and adolescents. J Nut 2007;137:153-7.

4. Cole TJ, Flegal KM, Nicholls D, Jackson AA. Body mass index cut offs to define thinness in children and adolescents: international survey. BMJ 2007;335:194.

5. Cole TJ, Bellizzi MC, Flegal KM, Dietz WH. Establishing a standard definition for child overweight and obesity worldwide: international survey. BMJ 2000;320:1240-3.

6. Wright C, Lakshman R, Emmett P, Ong KK. Implications of adopting the WHO 2006 Child Growth Standard in the UK: two prospective cohort studies. Arch Dis Child 2008;93:566-9.

7. Ferguson PW, Mitmesser SH, Maharaj N, Berseth CL. How is baby doing? Growth of US and Canadian infants using the Centers for Disease Control and Prevention and World Health Organization Charts. Nutr Today 2007;42:151-9.

8. van Buuren S, van Wouwe JP. WHO Child Growth Standards in action. Arch Dis Child 2008;93:549-51.

9. Schoeps DO, Leone C. Nutritional status and attained growth of preschool children attending Santo Andre (Brazil) daycare centers. Clin Nutr 2005;24:694 (Abstract). a sensibilidade, em detrimento da especificidade. Embora essa decisão possa ser uma política correta face à epidemia de excesso de peso que está ocorrendo em crianças, decorrente da Transição Nutricional, ela resulta em elevada frequência de falsos positivos, o que pode ocasionar uma sobrecarga aos serviços de atenção básica.

Obviamente, esses problemas só ocorrerão se o uso dos referenciais for feito de maneira rotineira, acrítica e isolada, esquecendo que o diagnóstico de crescimento e estado nutricional das crianças e dos adolescentes, individual ou coletivo, não deve ser elaborado apenas com base na antropometria, mas em conjunto com evidências clínicas e/ ou fatores socioambientais de risco, de modo a realizar um diagnóstico acurado.

Como conclusão, pode-se afirmar que a utilização dos novos referenciais resultará em maior prevalência de sobrepeso e obesidade, menor prevalência de magreza (desnutrição) em toda a idade pré-escolar e, ao mesmo tempo, numa menor prevalência de baixa estatura entre os maiores de cinco anos de idade.

10. Bertoli CJ, Leone C, Bertoli LM, Yamamoto J, Sakai SM. Crescimento de crianças de 3 a 6 anos de idade de creches públicas municipais de Taubate. Anais do $11^{\circ}$ Congresso Paulista de Pediatria; 2007 Mar 17-20; São Paulo, Brasil.

11. World Health Organization. Physical Status: the use and interpretation of anthropometry. Report of a WHO Expert Committee. Geneva: Switzerland; 1995.

12. Owen CG, Martin RM, Whincup PH, Smith GD, Cook DG. Effect of infant feeding on the risk of obesity across the life course: a quantitative review of published evidence. Pediatrics 2005;115;1367-77.

13. Martorell R, Stein AD, Schroeder DG. Early nutrition and later adiposity. J Nutr 2001;131:874S-80S.

14. Grummer-Strawn LM, Mei Z; Centers for Disease Control and Prevention Pediatric Nutrition Surveillance System. Does breastfeeding protect against pediatric overweight? Analysis of longitudinal data from the Centers for Disease Control and Prevention Pediatric Nutrition Surveillance System. Pediatrics 2004;113:e81-6.

15. Popkin BM, Richards MK, Montiero CA. Stunting is associated with overweight in children of four nations that are undergoing the nutrition transition. J Nutr 1996;126:3009-16.

16. Kain J, Uauy R, Lera L, Taibo M, Albala C. Trends in height and BMI of 6-year-old children during the nutrition transition in Chile. Obes Res 2005;13:2178-86.

17. Martorell R. Is wasting (thinness) a hidden problem in Latin America's children? J Nutr 2001;131:1133-4. 